\title{
Applications of Quantitative Microscopy in the Plastics Industry
}

\author{
Olivier Guise* and Carl Strom*
}

* SABIC Innovative Plastics, 1 Noryl Avenue, Selkirk, NY 12158

Conventional microscopy can be described as a process allowing the user to acquire a representative image of a sample. Conventional microscopy does not add value to the image itself beyond the 2D capture of a 3D object. In order to compare many images of similar samples one has to rely on a highly subjective visual evaluation to "rank" the features of interest within these images. As stated by Ian T. Young [1], conventional microscopy can be thought of as "the production of images that are to be used as images by human observers". By contrast quantitative microscopy can be thought of as "the production of images to be analyzed to produce data for human interpretation". Quantitative microscopy via image processing and analysis finds many applications in materials and polymer science. From the characterization of the size and shape of fillers to coating thickness measurements, it really changes the way we characterize materials in an industrial $R \& D$ environment. The benefits are many: a better understanding of our materials and of the relationship between their structure and their properties; an objective means of doing quality control and process control; a more rigorous approach to competitive materials analysis; quantitative and objective data for patent applications and customer support. Therefore, our goal here is to showcase examples of image processing and analysis for a variety of applications in the plastics industry, and to highlight their benefits in an R\&D setting. Some of these examples are listed below:

- Glass fibers are commonly used to increase strength and modulus in plastic materials. Their length can be significantly influenced by processing conditions and can be indicative of the "history" of the sample $[2,3]$. The ability to quantitatively measure the length distribution of glass fibers can be key to understanding the effect of the processing conditions on the materials.

- Transmission electron microscopy (TEM) offers remarkable resolution and is nowadays routinely used to investigate the morphology of materials down to the nanometer size and to answer questions such as dispersion of the fillers, nature and size distribution of the dispersed phase, etc. Combining TEM with image analysis adds a new dimension. This is especially true for instance to understand the level of exfoliation and the size of nanoclays in polymeric systems as a function of the processing conditions and the nature of the clays. This information can then be plugged into a modified Halpin-Tsai model to predict the system's entitlement.

- Scanning electron microscopy (SEM) obviously enjoys similar benefits when combined with image analysis and can be used to characterize the dispersed phase in various blends. This can provide information about the degree of compatibilization between the raw materials, which is directly influenced by the formulation and processing conditions. 


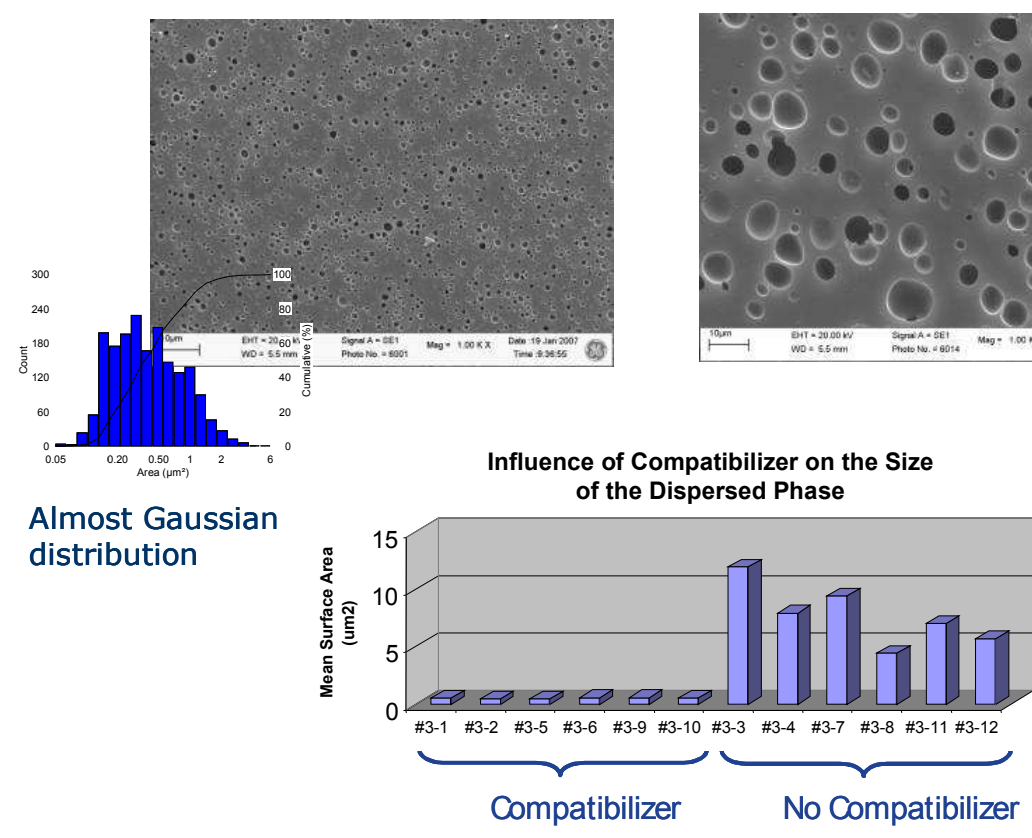

Figure 1: Application of quantitative microscopy to understand the influence of a compatibilizer on the morphology of an engineering thermoplastics material based on SEM images.

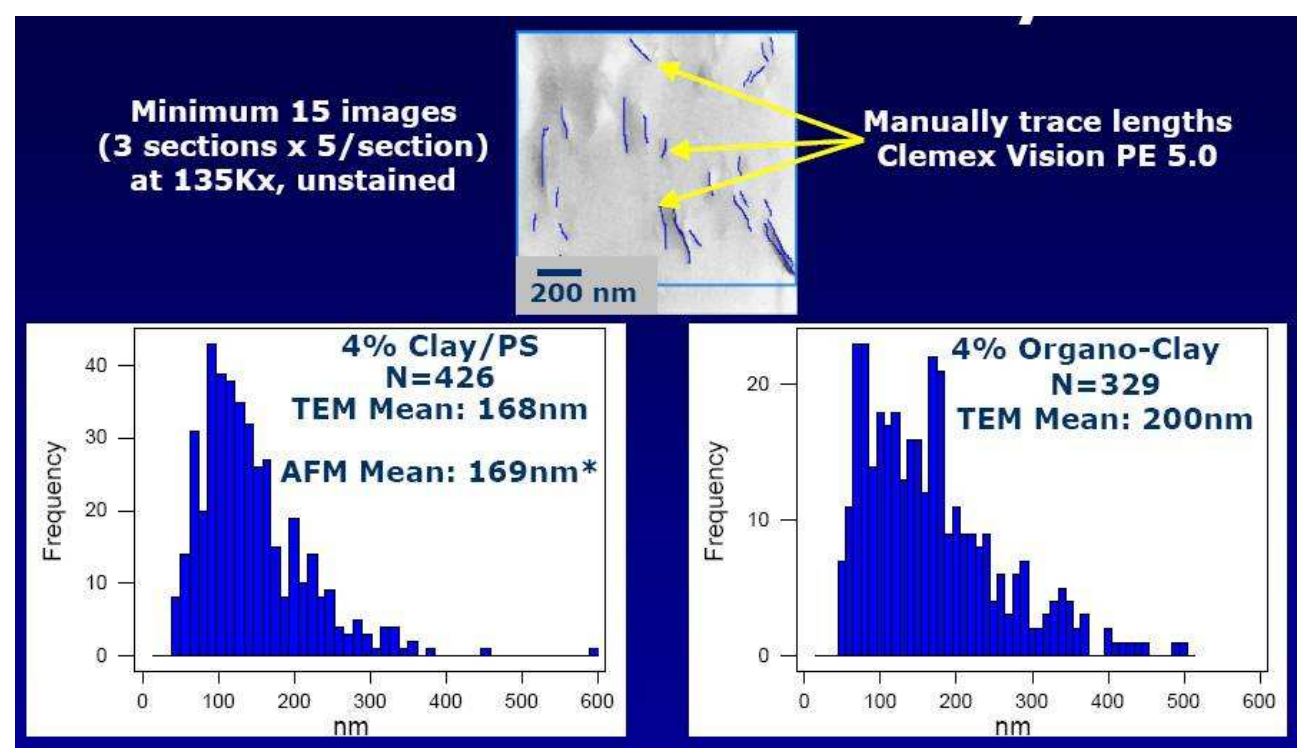

Figure 2: Analysis of the length distribution of nanoclays using TEM images.

\section{References}

1. Young, I.T., Quantitative Microscopy. 2006.

2. Laura, D.M., et al., Effect of glass fiber and maleated ethylene-propylene rubber content on tensile and impact properties of Nylon 6. Polymer, 2000. 41: p. 71657174.

3. Mlekusch, B., Fiber orientation in short-fiber-reinforced thermoplastics - Part II - Quantitative measurements by image analysis. Composites Science and Technology, 1999. 59: p. 547-560. 